\title{
Experimental examination of the variation of the spontaneous Brillouin power and frequency shift under the combined influence of temperature and strain
}

\author{
Mohammad Belal and Trevor P Newson \\ ORC, University of Southampton, Southampton, SO17 1BJ, United Kingdom
}

\begin{abstract}
We experimentally examine the validity of previous assumptions that the Brillouin coefficients describing the variation of the power of the spontaneous Brillouin and its frequency shift with respect to either strain or temperature are independent of the other parameter.

Keywords: Optical fibre sensors, Brillouin scattering, distributed temperature sensors

1. INTRODUCTION AND THEORY

Results of earlier attempts towards the characterization of the Brillouin frequency ${ }^{[1-2]}$ and its power to temperature and strain ${ }^{[3-4]}$ influences, validated it as a strong contender for optical fiber distributed temperature and strain sensing. Since then there have been numerous contributions to the field of distributed sensing exploiting the dependence of the Brillouin scattering to temperature and strain ${ }^{[5-10]}$. The layout of sensing rigs for Brillouin scattering based distributed sensing to date, have comprised separate sections of sensing fiber subjected to either temperature or strain influences. Interpretation of such experiments have been based on the premise that the Brillouin coefficients used to describe the influence of strain or temperature remain valid under circumstances where a sensing fiber is subjected to the combined influence of both temperature and strain. This paper for the first time experimentally investigates this premise.
\end{abstract}

1.1 The Brillouin coefficients

The change in Brillouin frequency and power due to strain and temperature has been represented by the matrix equation [5]

$\left[\begin{array}{l}\Delta v_{B} \\ \Delta I_{B_{A}, S}\end{array}\right]=\left[\begin{array}{ll}C_{B v}^{\varepsilon} & C_{B v}^{T} \\ C_{B I}^{\varepsilon} & C_{B I}^{T}\end{array}\right]\left[\begin{array}{l}\Delta \varepsilon \\ \Delta T\end{array}\right]$

where $C_{B v}^{\varepsilon}, C_{B v}^{T}$ are the Brillouin frequency coefficients and $C_{B I}^{\varepsilon}, C_{B I}^{T}$ are the Brillouin power coefficients for strain and temperature respectively, with $\Delta v_{B}$ and $\Delta I_{B_{A S S}}$ representing the change in peak Brillouin frequency and Brillouin anti-Stokes power with temperature and strain. Inverse of matrix equation 1 allows for values of strain $(\Delta \varepsilon)$ and temperature $(\Delta T)$ to be determined.

$\left[\begin{array}{c}\Delta \varepsilon \\ \Delta T\end{array}\right]=\frac{1}{\left|C_{B v}^{\varepsilon} C_{B I}^{T}-C_{B I}^{\varepsilon} C_{B v}^{T}\right|} \times\left[\begin{array}{cc}C_{B I}^{\varepsilon} & -C_{B v}^{T} \\ -C_{B I}^{T} & C_{B v}^{\varepsilon}\end{array}\right]\left[\begin{array}{l}\Delta v_{B} \\ \Delta I_{B_{A . S}}\end{array}\right]$

Equation 2 highlights that to estimate strain or temperature would require the usage of the Brillouin coefficients which so far have been measured by varying only one parameter, for example strain, whilst keeping the other constant i.e. temperature or vice versa, and the assumption has been that these coefficients are independent of the other parameter. This paper is the first attempt to experimentally investigate this.

\subsection{Brillouin frequency measurements}

Brillouin frequency measurements were made with the well established Brillouin optical correlation domain analysis (BOCDA) technique ${ }^{[8]}$. In BOCDA a frequency swept laser output is split into two. One of which is frequency shifted by an amount close to the expected Brillouin frequency shift, while the other beam remains un-shifted. Both beams are then launched into the opposite ends of the sensing fibre. A strong Brillouin interaction results in a localized region of the fibre corresponding to zero delay between the counter-propagating beams of light. The peak Brillouin frequency corresponding to this localised region is obtained by scanning the frequency of the frequency shifter. Delaying the phase of one beam relative to the other allows the position of the localised Brillouin interaction region to be swept along the 
fibre. This is normally achieved by incorporating a delay fiber outside the sensing fiber such that any change in the frequency at which the frequency of the laser is swept, translates into a change in phase and hence the sensing position along the sensing fiber.

The spatial resolution $(\Delta z)$ and the measurement range corresponding to the interval between correlation peaks $(\Delta d)$ are given by the following equations [8]:

$$
\Delta z=\frac{\Delta d . \Delta v_{B}}{\pi \cdot \Delta f} \quad ; \quad \Delta d=\frac{V_{g}}{2 f_{m}}
$$

where $f_{m}$ is the modulation frequency, $\Delta f$ is the frequency deviation achieved when the laser wavelength is swept at frequency $f_{m}, \Delta v_{B}$ is the linewidth of the Brillouin spectrum and $V_{g}$ is the velocity of light in the fibre. The above equations indicate that in this mode of operation, the ratio of spatial resolution to sensing length is determined by the ratio of the Brillouin gain linewidth compared to the modulation depth.

\subsection{Brillouin intensity measurements}

Brillouin intensity measurements were made using the Brillouin optical time domain reflectometry technique (B-OTDR). Laser pulses are launched into the sensing fibre and the interval between launched pulses is chosen to ensure scattering from a single pulse is obtained as it propagates through the sensing fibre. The backscattered Brillouin anti-Stokes signal is filtered and converted into an electrical signal through a photo receiver and displayed as a decay trace on the oscilloscope. The decay trace represents the change in Brillouin anti-Stokes power as a function of distance along the sensing fibre. The required bandwidth of the electronics and the pulse width are defined by the desired spatial resolution.

\section{EXPERIMENTAL SETUP AND PROCEDURE}

2.1 Experimental Setup and procedure for Brillouin frequency measurement

The experimental set up for Brillouin frequency measurements is shown in figure 1.
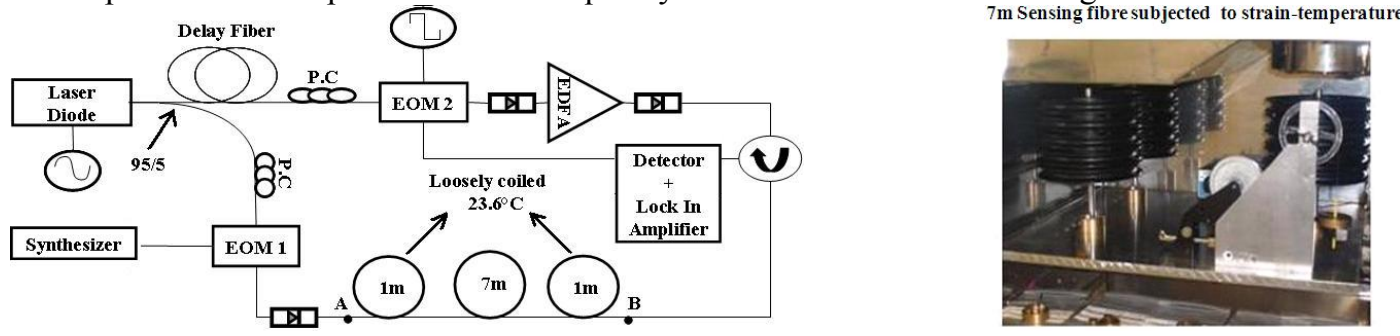

Figure1. Experimental configuration for the Brillouin frequency measurements and the inside of the Environmental chamber showing the arrangement for straining the $7 \mathrm{~m}$ section of sensing fiber using weights

The $7 \mathrm{~m}$ of sensing fiber comprised standard single mode fiber with an effective area $\sim 80 \mu \mathrm{m}^{2}$, loss $\sim 0.20 \mathrm{~dB} / \mathrm{km}$ and dispersion $\sim 17 \mathrm{ps} / \mathrm{km} \mathrm{nm}$ at $1550 \mathrm{~nm}$. It was arranged on a strain rig consisting of pulleys inside an environmental chamber, as shown in figure 1 . The remaining sections of $1 \mathrm{~m}$ each were loosely coiled and maintained at $23.6^{\circ} \mathrm{C}$ outside the environmental chamber. The injection current of the laser diode (linewidth $\sim 5 \mathrm{MHz}, 1550 \mathrm{~nm}$ ) was modulated to achieve a frequency deviation $\Delta f$ of $8 \mathrm{GHz}$ with sinusoidal sweep rates $f_{m}$ of $74.4 \mathrm{kHz}$. EOM 1 was driven by a synthesizer to generate side bands corresponding to the expected Brillouin frequency shift of $\sim 10-12 \mathrm{GHz}$. A signal generator was used to provide a $30 \mathrm{kHz}$ (square wave) signal to the EOM 2 which also serves as a reference signal to the lock-in amplifier with bandwidth of $100 \mathrm{kHz}$. The Brillouin signal was detected using a $1 \mathrm{GHz}$ detector with responsitivity of $0.95 \mathrm{~A} / \mathrm{W}$.

2.2 Experimental Setup and procedure for the Brillouin intensity measurement Experimental set up for Brillouin intensity measurements shown below along with detail of reference and sensing fiber. 


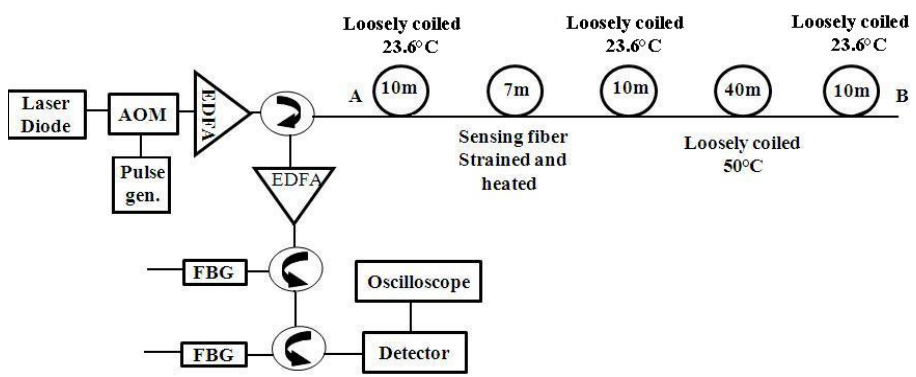

Figure2. Experimental configuration for the Brillouin intensity measurements

To achieve an adequate signal to noise, a pulse width of $30 \mathrm{~ns}$ was used, corresponding to a spatial resolution of $3 \mathrm{~m}$. To accommodate this increased spatial resolution the fibre either side of the $7 \mathrm{~m}$ sensing section was increased from $1 \mathrm{~m}$ to $10 \mathrm{~m}$. An additional $40 \mathrm{~m}$ long loosely coiled fibre was added to the sensing fibre and maintained at $50^{\circ} \mathrm{C}$ to facilitate tuning the FBG filters to the centre of the Brillouin frequency shift. The output of the laser diode (linewidth $\sim 5 \mathrm{MHz}$, $1533.95 \mathrm{~nm}$ ) was pulsed using an acousto-optic modulator to achieve 30ns rectangular shaped optical pulses at a repetition rate of $1 \mathrm{MHz}$, which were amplified to peak powers of $1 \mathrm{~W}$ before being launched into the sensing fiber. The backscattered Brillouin anti-Stokes signal was amplified and filtered using two cascaded tunable narrow fiber Bragg gratings ( $3.5 \mathrm{GHz}$ band width) before detection. The backscattered Brillouin anti-Stokes traces for varying temperatures at fixed values of strain $(274.7 \mu \varepsilon-1463.3 \mu \varepsilon)$ were normalised using the Brillouin anti-Stokes traces collected at room temperature for each applied strain. Traces normalised in this manner were used to calculate the Brillouin intensity coefficient for temperature at different values of strain $(274.7 \mu \varepsilon-1463.3 \mu \varepsilon)$. Similarly the backscattered Brillouin antiStokes traces for varying strain at fixed values of temperature $\left(29.8^{\circ} \mathrm{C}-69.5^{\circ} \mathrm{C}\right)$ were normalised using the backscattered Brillouin anti-Stokes traces collected at $0 \mu \varepsilon$ for each temperature. These normalised traces were used to estimate the Brillouin intensity coefficient for strain at different values of temperature $\left(29.8^{\circ} \mathrm{C}-69.5^{\circ} \mathrm{C}\right)$.

\section{RESULTS AND DISCUSSION}

\subsection{Results of Brillouin frequency and intensity measurement}
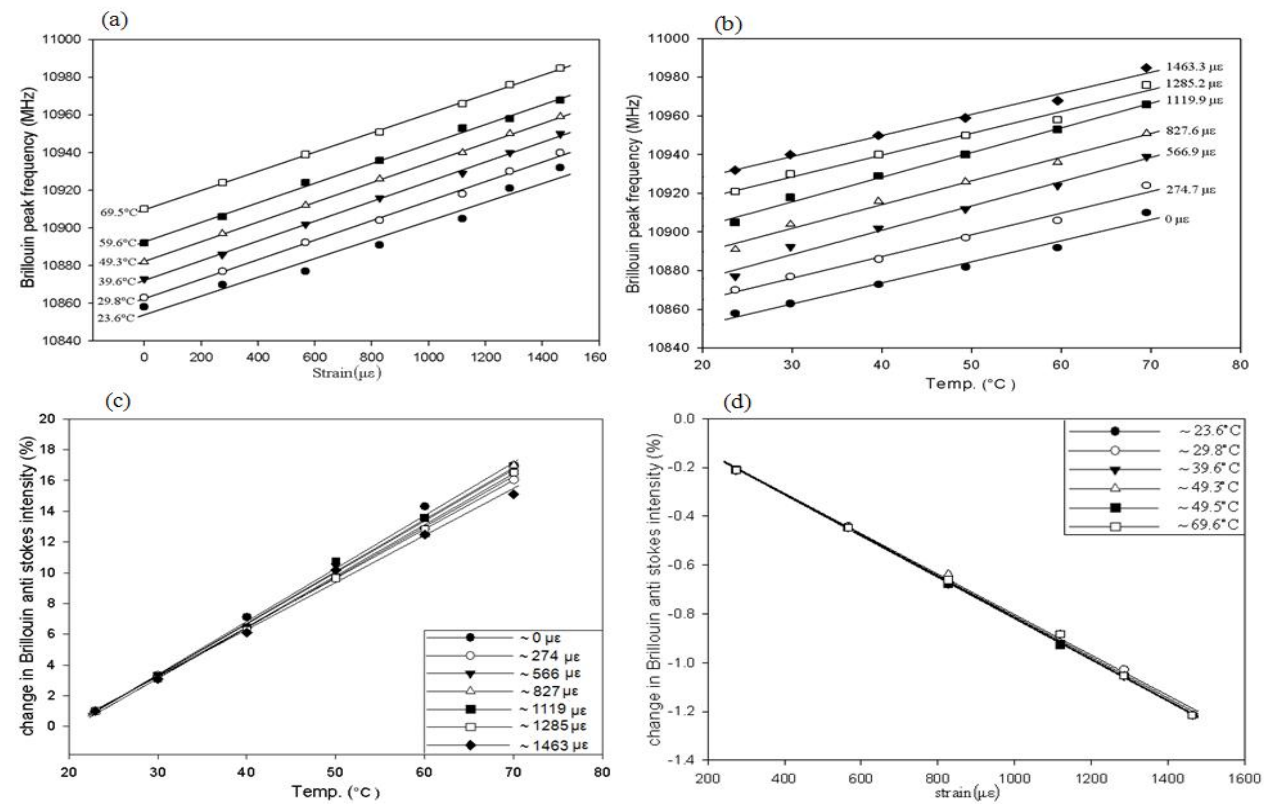

Figure 3(a) Changes in peak Brillouin frequency with strain at different temperatures; (b) Changes in peak Brillouin frequency with temperature at different strains; (c) The percentage change in Brillouin anti Stokes power with temperature at different strains; (d) The percentage change in Brillouin anti Stokes power with strain at different temperatures

Plots in figure 3(a), show the shifts in peak Brillouin frequency with applied strain at different temperatures and plots in figure 3(b), show the shifts in peak Brillouin frequency with temperature at different values of strain. Similarly the plots in figure 3(c), show the percentage change in Brillouin anti Stokes power with temperature at different values of strain, 
while those in figure 3(d), depict percentage changes in Brillouin anti Stokes intensity with strain at different temperatures. Using the data on glass characterization ${ }^{[11]}$, a correction of $0.5 \%(0.2 \mu \varepsilon-1.4 \mu \varepsilon)$ was made to the theoretically estimated values of Young's moduli over a temperature range of $23.6^{\circ} \mathrm{C}$ to $69.5^{\circ} \mathrm{C}$.

\subsection{Summary of Results}

Figures 4 (a), (b) show the variation in the Brillouin coefficients of frequency with temperature and strain for different strains and temperatures respectively. Likewise figures 4 (c), (d) show the variation of the Brillouin coefficients of power with temperature and strain for different strains and temperatures respectively. The error bars depict 1 standard deviation.
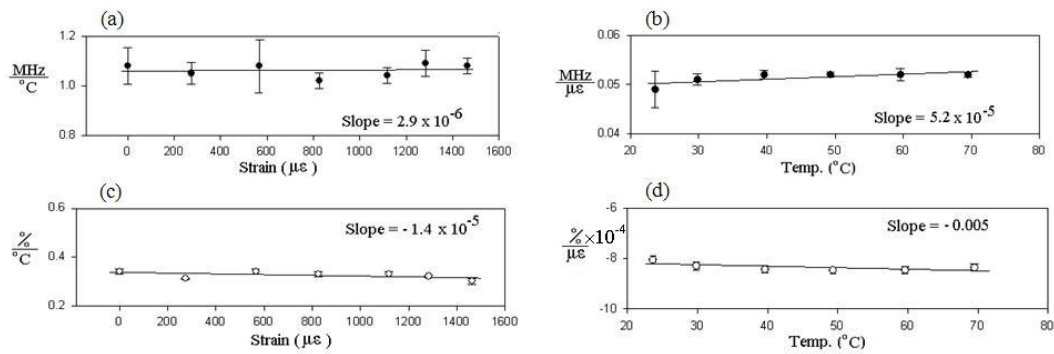

Figure 4(a) Brillouin frequency coefficient for temperature at different values of strain; (b) Brillouin frequency coefficient for strain at different values of temperature; (c) Brillouin intensity coefficient for temperature at different values of strain; (d) Brillouin intensity coefficient for strain at different values of temperature

Considering a specific case of simultaneously imposed strain of $1285 \mu \varepsilon$ and a temperature of $70^{\circ} \mathrm{C}$, on a section of sensing fibre, a $118 \mathrm{MHz}$ and $15.51 \%$ change in Brillouin frequency and power is observed experimentally for this section with reference to loosely coiled fiber maintained at room temperature. Using the value of Brillouin coefficients at $23.6^{\circ} \mathrm{C}$ and $0 \mu \varepsilon$, calculated from figure 4 , in equation 2 the values of simultaneously applied strain and temperature are estimated to be $1295 \mu \varepsilon$ and $73.8^{\circ} \mathrm{C}$ respectively, as compared to the applied strain of $1285 \mu \varepsilon$ and temperature of $70^{\circ} \mathrm{C}$.

\section{CONCLUSIONS}

Although the slopes in figures 4 (a) - (d) show variation of Brillouin frequency and intensity coefficients, under combined temperature and strain influences over the range of temperature and strain experimentally investigated, analysis demonstrates that the slope of figure 4 (c) is the dominant factor responsible for a $3.8^{\circ} \mathrm{C}$ error in temperature measurement. For accurate measurements one must take into account the variation of the Brillouin intensity coefficient with temperature as the applied strain is varied.

\section{REFERENCES}

[1] Horiguchi T, Kurashima $\mathrm{O}$ and Tateda M, "Tensile strain dependence of Brillouin frequency shift in silica optical fibers", IEEE Photon. Technol. Lett., 1(5), 107-108 (1989)

[2] Kurashima T, Horiguchi T, and Tateda M, "Thermal effects on the Brillouin frequency shift in jacketed optical silica fibers” Applied Optics, 29(15), 2219 - 2222 (1990)

[3] Wait PC, Newson T P, "Landau Placzek ratio applied to distributed fibre sensing”, Opt. Comms., 122, 141-6 (1996)

[4] DeSouza K, Wait P C, Newson T P, "Characterisation of strain dependence of the Landau-Placzek ratio for distributed sensing", Elect. lett., 33(7), 615-616 (1997)

[5] Parker T R, Farhadiroushan M, Handerek V A and Rogers A J, “Temperature and strain dependence of the power level and frequency of spontaneous Brillouin scattering in optical fibers", Opt. Lett., 22(11), 787 - 789 (1997)

[6] Kee H H, Lees G P, Newson T P, "An all-fiber system for simultaneous interrogation of distributed strain and temperature sensing using spontaneous Brillouin scattering”, Opt. Lett,. 25(10), 695-697 (2000)

[7] Maughan S M, Kee H H and Newson T P, "Simultaneous distributed fibre temperature and strain sensor using microwave coherent detection of spontaneous Brillouin backscatter", Meas. Sci. Technol., 12, 834-842 (2001)

[8] Song K Y, He Z and Hotate K, "Distributed strain measurement with millimeter-order spatial resolution based on Brillouin optical correlation domain analysis", Opt. Lett., 31(17), 2526-2528 (2006)

[9] Belal M, Cho Y T, Ibsen M and Newson T P “A temperature compensated high spatial resolution distributed strain sensor", Meas. Sci. Technol., 21 (2010)

[10] Soto M A, Bolognini G, Pasquale F D, and Thévenaz L, "'Simplex-coded BOTDA fiber sensor with $1 \mathrm{~m}$ spatial resolution over a $50 \mathrm{~km}$ range", Opt. Lett., 35(2), 259 - 261 (2010)

[11] Bansal N P and Doremus R H, "Handbook of glass properties chapter 2 Academic press" (1986) 